\title{
ON THE DEVELOPMENT OF CONTINUOUS FUNCTIONS IN SERIES OF TCHEBYCHEFF POLYNOMIALS*
}

BY

\section{J. A. SHOHAT (JACQUES CHOKHATE)}

Introduction. Consider a system of orthogonal and normal Tchebycheff polynomials

$$
\varphi_{n}(x)=a_{n} x^{n}+\cdots \quad\left(n=0,1,2, \cdots ; a_{n}>0\right)
$$

corresponding to a certain interval $(a, b)$ with the characteristic function $p(x)$ integrable and not negative on $(a, b)$. Thus we have

$$
\int_{a}^{b} p(x) \varphi_{m}(x) \varphi_{n}(x) d x= \begin{cases}0, & m \neq n, \\ 1, & m=n .\end{cases}
$$

For any function $f(x)$ we have a formal development as follows:

$$
f(x) \sim \sum_{0}^{\infty} A_{i} \varphi_{i}(x), \quad A_{i}=\int_{a}^{b} p(x) f(x) \varphi_{i}(x) d x,
$$

provided, of course, the right hand integrals exist.

Let us write

$$
f(x)=\sum_{0}^{n} A_{i} \varphi_{i}(x)+r_{n, f}(x) \equiv P_{n, f}(x)+r_{n, f}(x) .
$$

The question arises as to the convergence of the development (I) to $f(x)$ or-what is the same-the behavior of $r_{n, f}(x)$ in (II) for $n \rightarrow \infty$.

The case $(a, b)=(-1,1), p(x)=1$ leads to Legendre's polynomials; it has been treated by Professor D. Jackson.t

In this paper we follow the method given by Professor Jackson in order to investigate the convergence of the development (I) involving Tchebycheff's polynomials in general. Hereafter, the interval $(a, b)$ is supposed to be finite, and $f(x)$ to be continuous on $(a, b)$.

1. $r_{n, f}(x)$ expressed as a definite integral. We obtain easily, using the formulas for $A_{i}$,

$$
r_{n, f}(x)=f(x)-\int_{a}^{b} p(y) f(y) \sum_{0}^{n} \varphi_{i}(x) \varphi_{i}(y) d y,
$$

* Presented to the Society, April 19, 1924.

$\dagger \mathrm{D}$. Jackson, On the degree of convergence of a continuous function according to Legendre's polynomials, these Transactions, vol. 13 (1912), pp. 305-318. 
which gives, for $f(x) \equiv 1$,

$$
1=\int_{a}^{b} p(y) \sum_{0}^{n} \varphi_{i}(x) \varphi_{i}(y) d y \text {. }
$$

Hence,

$$
\begin{gathered}
r_{n, f}(x)=\int_{a}^{b} p(y)[f(x)-f(y)] K_{n}(x, y) d y, \\
K_{n}(x, y)=\sum_{0}^{n} \varphi_{i}(x) \varphi_{i}(y) \\
r_{n, f}(x)=\frac{a_{n}}{a_{n+1}} \int_{a}^{b} p(y)[f(x)-f(y)] \frac{\varphi_{n+1}(x) \varphi_{n}(y)-\varphi_{n}(x) \varphi_{n+1}(y)}{x-y} d y,
\end{gathered}
$$

since

$$
K_{n}(x, y)=\frac{a_{n}}{a_{n+1}} \frac{\varphi_{n+1}(x) \varphi_{n}(y)-\varphi_{n}(x) \varphi_{n+1}(y)}{x-y} .^{*}
$$

Following Jackson's method we get for any polynomial $Q_{n}(x)$, of degree $\leqq n$,

$$
\begin{aligned}
0 & =\int_{a}^{b} p(y)\left[Q_{n}(x)-Q_{n}(y)\right] K_{n}(x, y) d y, \\
r_{n, f}(x) & =\int_{a}^{b} p(y)[\varphi(x)-\varphi(y)] K_{n}(x, y) d y, \\
\varphi(x) & \equiv f(x)-Q_{n}(x) .
\end{aligned}
$$

We substitute here for $Q_{n}(x)$ a special polynomial, namely the polynomial $T_{n, f}(x)$ of best approximation to $f(x)$ on $(a, b)$ (of degree $n$ ). Thus we get two formulas for $r_{n, f}(x)$ :

$$
\begin{aligned}
\text { (1) } \quad r_{n, f}(x)= & \int_{a}^{b} p(y)[\varphi(x)-\varphi(y)] K_{n}(x, y) d y \\
& K_{n}(x, y)=\sum_{0}^{n} \varphi_{i}(x) \varphi_{i}(y) ; \quad \varphi(x) \equiv f(x)-T_{n, f}(x) ; \\
\text { (2) } \quad r_{n, f}(x)= & \frac{a_{n}}{a_{n+1}} \int_{a}^{b} p(y)[\varphi(x)-\varphi(y)] \frac{\varphi_{n+1}(x) \varphi_{n}(y)-\varphi_{n}(x) \varphi_{n+1}(y)}{x-y} d y .
\end{aligned}
$$

Denote by $E_{n}(f)$ the best approximation on $(a, b)$ of $f(x)$ by means of a polynomial of degree $n$, i. e.

$$
E_{n}(f)=\max \left|f(x)-T_{n, f}(x)\right| \text { for } a \leqq x \leqq b .
$$

* Darboux, Mémoire sur l'approximation des fonctions de très grands nombres, Jo u rnal de Mathématiques Pures et Appliquées, ser. 3, vol. 4 (1878), pp. 5-60, 377-416; p. 413. 
Using Schwarz's inequality, we get from (1)

$$
\begin{aligned}
\left|r_{n, f}(x)\right| & \leqq 2 E_{n}(f) \sqrt{\int_{a}^{b} p(y) d y} \sqrt{\int_{a}^{b} p(y) K_{n}^{2}(x, y) d y} \\
& =2 E_{n}(f) \sqrt{\int_{a}^{b} p(y) d y} \sqrt{\sum_{0}^{n} \varphi_{i}^{2}(x)}, \\
\left|r_{n, f}(x)\right| & \leqq 2 Q E_{n}(f) \sqrt{K_{n}(x)} \quad\left(Q^{2}=\int_{a}^{b} p(y) d y\right), \\
K_{n}(x) & \equiv K_{n}(x, x)=\sum_{0}^{n} \varphi_{i}^{2}(x) .
\end{aligned}
$$

$E_{n}(f)$, as a function of $n$, has been investigated by Lebesgue, de la ValléePoussin, S. Bernstein, W. Stekloff, and in particular by D. Jackson.*

Table A: $E_{n}(f)$

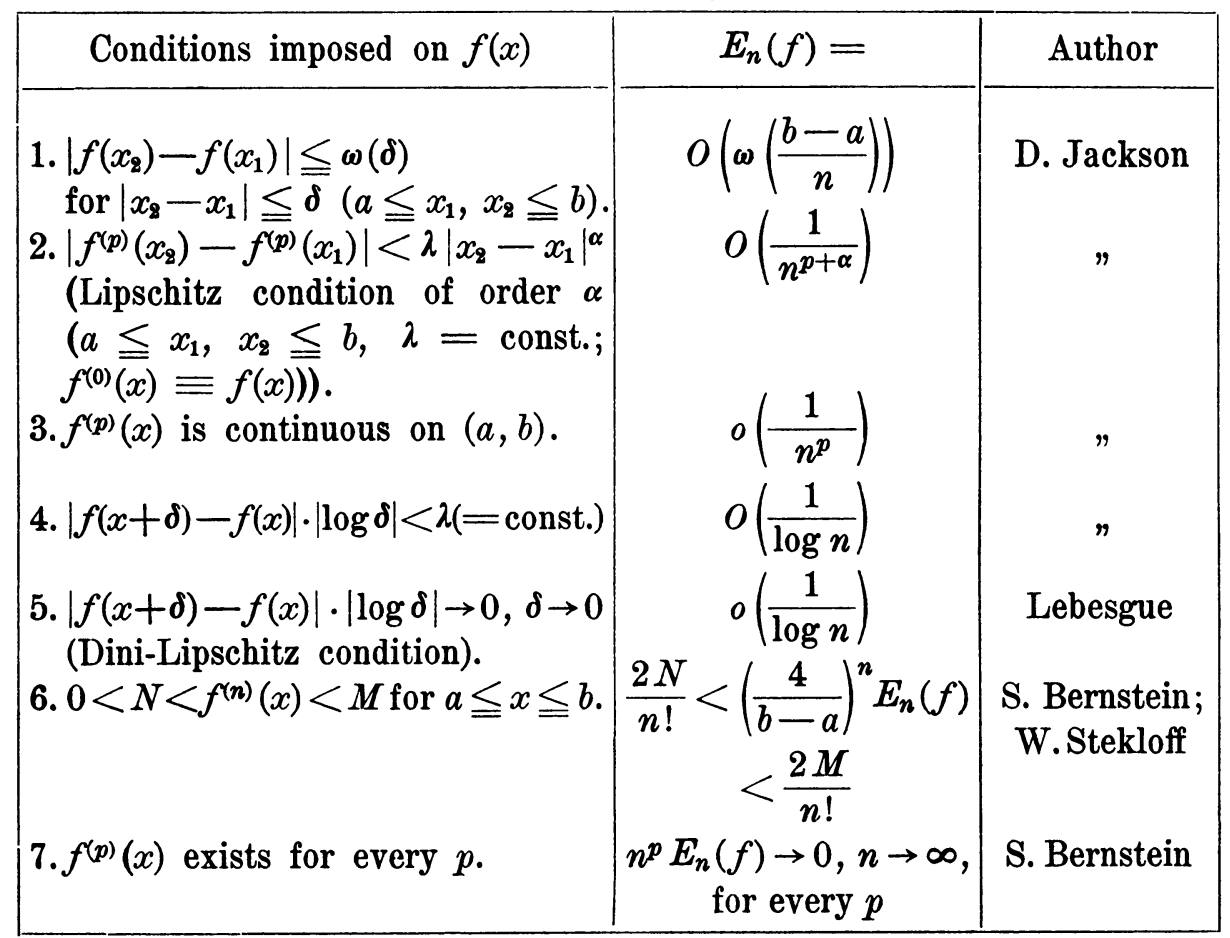

We see from Table A that in order to evaluate $r_{n, f}(x)$ by means of $(1,2,4)$, we need to know the order of $\varphi_{n}(x)$ or $K_{n}(x)$ with respect to $n$.

* D. Jackson, Ubber die Genauigkeit der Annäherung stetiger Funktionen, Dissertation, Göttingen, 1911, pp. 1-96. 
2. Order of $K_{n}(x)$ (with respect to $n$ ). It can be proved easily that

$$
\frac{1}{K_{n}(z)}=\min \int_{a}^{b} p(y)\left[1+Z_{1}(y-z)+\cdots+Z_{n}(y-z)^{n}\right]^{2} d y^{*}
$$

Therefore, using the notation $K_{n}(p ; z)$, we conclude that

$$
\begin{aligned}
& p_{2}(x) \geqq p(x) \geqq p_{1}(x) \text { for } a \leqq x \leqq b \text { implies } \\
& K_{n}\left(p_{2} ; z\right) \leqq K_{n}(p ; z) \leqq K_{n}\left(p_{1} ; z\right) .
\end{aligned}
$$

On the other hand, to the characteristic function

$$
\begin{aligned}
& p_{1}(x)=(x-a)^{\alpha-1}(b-x)^{\beta-1} \Pi(x) \quad(\alpha, \beta>0), \\
& \Pi(x) \text { a polynomial }(\Pi(a) \Pi(b) \neq 0),
\end{aligned}
$$

there corresponds a special system of Tchebycheff's polynomials, a generalization of Jacobi's polynomials $(\Pi(x) \equiv 1)$, and I have obtained the asymptotic expression for $K_{n}\left(p_{1} ; z\right)$ at any point $z$ in $(a, b)$ t.

Thus we have

$$
\begin{gathered}
K_{n}\left(p_{1} ; a\right) \sim n^{2 \alpha}, \quad K_{n}\left(p_{1} ; b\right) \sim n^{2 \beta}, \\
K_{n}\left(p_{1} ; z\right) \sim n^{2 m+1},
\end{gathered}
$$

$z$ being a root of $\Pi(x)$ of multiplicity $2 m \geqq 0(a+\varepsilon \leqq z \leqq b-\varepsilon)_{+}^{+}$. These results enable us to prove the following

THeorem I. (i) Suppose the point $x=z$ be inside the interval $(a, b)$ : $a+\varepsilon \leqq z \leqq b-\varepsilon$, and that there exist finite numbers $k>-1, A>0, c, d$ such that

$$
\frac{p(x)}{|x-z|^{k}} \geqq A \text { for }(a \leqq) c \leqq x \leqq d(\leqq b) \quad(c<z<d) .
$$

Let us take the smallest $k$ possible satisfying the above conditions and $k=0$ in case $p(z)>0$. Then $K_{n}(p ; z)=O\left(n^{2 k^{\prime}+1}\right)$, where $k^{\prime}$ is the smallest integer $\geqq k / 2$. In particular $K_{n}(p ; z)=O(n)$ for $k \leqq 0$.

(ii) Suppose the point $x=z$ coincides with one of the end points of $(a, b)$, say $z=a$. If

$$
\frac{p(x)}{|x-a|^{k}} \geqq A \quad(k>-1, A>0 ; a \leqq x \leqq c(\leqq b)),
$$

\footnotetext{
* See my paper (where the proof is given for $z=0$ ), Jacques Chokhate, Sur le développement de l'intégrale $\int_{a}^{b}[p(y) /(x-y)] d y$ en fraction continue et sur les polynomes de Tchebycheff, Rendiconti del Circolo Matematico di Palermo, vol. 47 (1923), pp. $25-46 ;$ p. 41.

† On the asymptotitc properties of a certain class of Tchebycheff's polynomials, read before the International Mathematical Congress, Toronto, August, 1924.

$\ddagger$ Hereafter $\varepsilon$ stands for an arbitrarily small but fixed quantity.
} 
then

$$
K_{n}(p ; a)=O\left(n^{2 k+2}\right) .
$$

Proof. (i) Consider the characteristic function corresponding to the interval $(c, d)$ and defined as follows:

$$
p_{1}(x)=A^{\prime}(x-z)^{2 k^{\prime}} \text { in }(c, d) \quad\left(A^{\prime}>0 ; c<z<d\right) .
$$

We have, then, $A^{\prime}$ being sufficiently small,

$$
p(x) \geqq p_{1}(x) \text { for } c \leqq x \leqq d .
$$

Therefore (see 7,9), since

$$
\begin{gathered}
\min \int_{a}^{b} p(y)\left[1+Z_{1}(y-z)+\cdots+Z_{n}(y-z)^{n}\right]^{2} d y \\
\geqq \min \int_{c}^{d} p(y)\left[1+Z_{1}(y-z)+\cdots+Z_{n}(y-z)^{n}\right]^{2} d y, \\
K_{n}(p ; z) \leqq K_{n}\left(p_{1} ; z\right)=O\left(n^{2 k^{\prime}+1}\right),
\end{gathered}
$$

In a similar, slightly modified, way we prove the statement (ii) of our theorem. Formula (4) leads to the following

COROLlary. If $p(x)$ satisfies the conditions of Theorem I, then

$$
\begin{aligned}
& \left|r_{n, f}(z)\right|<\tau E_{n}(f) n^{k^{\prime}+1 / 2} \quad(a+\varepsilon \leqq z \leqq b-\varepsilon), \\
& \left|r_{n, f}(z)\right|<\tau E_{n}(f) n^{k+1} \quad((z-a)(z-b)=0) .^{*}
\end{aligned}
$$

3. Order of $r_{n, f}(x)$ (with respect to $n$ ) (method of D. Jackson). For any system of Tchebycheff's polynomials the following inequality holds:

$$
\frac{a_{n}}{a_{n+1}}<\frac{b-a}{2} .+
$$

Consider two cases:

Case I. The point $x=z$ is inside the interval $(a, b): a<c<z<d<b$. We write $(1,2)$ as follows:

$$
\begin{gathered}
r_{n, f}(z)=\int_{a}^{c+\varepsilon}+\int_{c+\varepsilon}^{z-\varepsilon_{n}}+\int_{z-\varepsilon_{n}}^{z+\varepsilon_{n}}+\int_{z+\varepsilon_{n}}^{d-\varepsilon}+\int_{d-\varepsilon}^{b}=i_{1}+i_{2}+i_{3}+i_{4}+i_{5}, \\
\varepsilon_{n}>0, \quad \varepsilon_{n} \rightarrow 0 \text { for } n \rightarrow \infty .
\end{gathered}
$$

* Hereafter we use $\tau$ to denote generally a fixed positive quantity, different, of course in different formulas, which does not depend on $n$.

† J. Chokhate, loc. cit., p. 33. 
Here $\varepsilon$ denotes a certain fixed positive quantity; $n$ is supposed to be so large and $\varepsilon$ so small that we have

$$
\varepsilon_{n}<\varsigma, \quad c+2 \varepsilon \leqq z \leqq d-2 \varepsilon .
$$

Suppose the system of Tchebycheff's polynomials under consideration subjected to following conditions:

$$
\left.\begin{array}{c}
p(x)<P(=\text { fixed const. }) \\
\left|\varphi_{n}(x)\right|<\tau n^{\sigma} \quad\left(n=1,2, \ldots ; \sigma>-\frac{1}{2}^{*}\right)
\end{array}\right\}(c \leqq x \leqq d),
$$

where $P, \tau, \sigma$ do not depend upon $x$, nor upon $n$.

Consider first $i_{1}$ and $i_{5}$ in (11). Here we use formula (2), since $1 /|x-y|<\varepsilon$. Using $(3,10)$ we get

$$
\begin{gathered}
\left|i_{1}\right|,\left|i_{5}\right|<\frac{b-a}{2} E_{n}(f)\left\{\left|\varphi_{n+1}(z)\right| \int_{a}^{b} p(y)\left|\varphi_{n}(y)\right| d y+\left|\varphi_{n}(z)\right| \int_{a}^{b} p(y)\left|\varphi_{n+1}(y)\right| d y\right\} \\
(14) \quad\left|i_{1}\right|,\left|i_{5}\right|<\tau E_{n}(f) n^{\sigma}
\end{gathered}
$$

assuming only the condition $\left|\varphi_{n}(z)\right|<\tau n^{\sigma}$ (since

$$
\int_{a}^{b} p(y)\left|\varphi_{i}(y)\right| d y<\sqrt{\int_{a}^{b} p(y) d y}
$$

by Schwarz's inequality). We use the same formula (2) to estimate $i_{z}$ and $i_{4}$.

Putting $z-y=u$, we get

$$
\begin{gathered}
\left|i_{2}\right|,\left|i_{4}\right|<\tau n^{2 \sigma} E_{n}(f) \int_{\varepsilon_{n}}^{b-a} \frac{d u}{u} \\
\left|i_{2}\right|,\left|i_{4}\right|<\tau n^{2 \sigma} E_{n}(f)\left|\log \varepsilon_{n}\right|
\end{gathered}
$$

under conditions $(12,13)$. In order to estimate $i_{3}$ in $(11)$, we write

which gives

$$
i_{3}=\int_{z-\varepsilon_{n}}^{z+\varepsilon_{n}} p(y)[\varphi(z)-\varphi(y)] \sum_{0}^{n} \varsigma_{i}(z) \varphi_{i}(y) d y
$$

$$
\begin{gathered}
\left|i_{3}\right|<\tau E_{n}(f)\left[\varphi_{0}^{2}+\sum_{1}^{n} i^{2 \sigma}\right] \int_{z-\varepsilon_{n}}^{z+\varepsilon_{n}} p(y) d y \\
\left|i_{3}\right|<\tau E_{n}(f) n^{2 \sigma+1} \varepsilon_{n}
\end{gathered}
$$

under conditions $(12,13)$. If we replace condition (13) by a less restrictive one, 


$$
\left|\varphi_{n}(z)\right|<\tau n^{\sigma} \quad\left(n=1,2, \cdots ; \sigma>-\frac{1}{2}\right),
$$

we can estimate $i_{2}, i_{4}$ and $i_{3}$ as follows:

Apply Schwarz's inequality t $i_{2}, i_{4}$ in (11). We get, since here $1 /|z-y| \leqq 1 / \varepsilon_{n}$,

$$
\left|i_{2}\right|,\left|i_{4}\right|<\tau \frac{E_{n}(f)}{\varepsilon_{n}} n^{\sigma}
$$

assuming only the condition (18). Assuming two conditions, (12) and (18), we get

$$
\begin{gathered}
\left|i_{2}\right|<v E_{n}(f) \sqrt{\int_{a+\varepsilon}^{z-\varepsilon_{n}} \frac{d y}{(z-y)^{2}}} \sqrt{\varphi_{n+1}^{2}(z)+\varphi_{n}^{2}(z)} ;^{*} \\
\left|i_{8}\right|,\left|i_{4}\right|<\boldsymbol{\frac { E _ { n } ( f ) } { V }} n^{\sigma} .
\end{gathered}
$$

Similarly, applying Schwarz's inequality to $i_{3}$ in (16), we get

$$
\begin{aligned}
& \left|i_{3}\right|<\tau E_{n}(f) \sqrt{\int_{z-\varepsilon_{n}}^{z+\varepsilon_{n}} p(y) d y} \sqrt{\int_{a}^{b} p(y) K_{n}^{2}(z, y) d y} \\
& \left|i_{3}\right|<\tau E_{n}(f) \sqrt{\varepsilon_{n}} \sqrt{K_{n}(z)} \text { (under conditions (12)); } \\
& \left|i_{3}\right|<\tau E_{n}(f) \sqrt{\varepsilon_{n}} n^{\sigma+1} \quad \text { (under conditions }(12,18) \text { ). }
\end{aligned}
$$

Case II. $(z-a)(z-b)=0$; say $z=b$. Assume, as above,

$$
\left.\begin{array}{c}
p(x)<P \\
\left|\varphi_{n}(x)\right|<v n^{\sigma} \quad\left(n=1,2, \ldots ; \sigma>-\frac{1}{2}\right)
\end{array}\right\}((a \leqq) c \leqq x \leqq b),
$$

$$
\left|\varphi_{n}(b)\right|<v n^{\sigma} \quad\left(n=1,2, \ldots ; \sigma>-\frac{1}{2}\right) .
$$

Write $(1,2)$ as follows:

$$
\begin{gathered}
r_{n, f}(b)=\int_{a}^{c+\varepsilon}+\int_{c+\varepsilon}^{b-\varepsilon_{n}}+\int_{b-\varepsilon_{n}}^{b}=i_{1}+i_{2}+i_{3}, \\
\varepsilon_{n}>0, \quad \varepsilon_{n} \rightarrow 0 \text { for } n \rightarrow \infty ; \varepsilon>0 ; \quad \varepsilon_{n}<\varepsilon ; \quad c+z \varepsilon<b .
\end{gathered}
$$

Following the preceding discussion we estimate $i_{1}, i_{2}, i_{3}$ in a manner quite similar to that given above and find similar results.

We proceed to specify the infinitesimal $\varepsilon_{n}$. Take $\varepsilon_{n}=n^{-\beta}$, with $\beta>0$, and choose $\beta$ so as to make $r_{n, f}(z)$ of the highest order possible with respect to $1 / n$. The results thus found (using the expressions above for $\left.i_{1}, i_{2}, i_{3}, i_{4}, i_{5}\right)$ are summarized in the following table.

* Similar inequality for $\left|i_{4}\right|$. 
Table B: $r_{n, f}(x)$

\begin{tabular}{|c|c|c|c|c|}
\hline \multirow{2}{*}{ Case } & \multicolumn{3}{|c|}{ Conditions imposed on $p(x), \varphi_{n}(x)$} & \multirow{2}{*}{$\begin{array}{c}\left|r_{n, f}(z)\right|<\tau E_{n}(f) h_{n}{ }^{*} \\
\text { with } h_{n}=\end{array}$} \\
\hline & $p(x)$ is & $\varphi_{n}(x)<\tau n^{\sigma}$ for & with $\sigma$ & \\
\hline 1. $a<c<z<d<b$ & $\begin{array}{c}\text { bounded } \\
\text { for } c \leqq x \leqq d\end{array}$ & $c \leqq x \leqq d$ & $<0$ & $\begin{array}{c}n^{\sigma} \text { (impossible; see } \\
\text { below) }\end{array}$ \\
\hline 2. & $\bar{n}$ & $"$ & $0 \leqq \sigma<\frac{1}{4}$ & $n^{2 \sigma} \log n$ \\
\hline 3. & $"$ & " & $\sigma \geqq \frac{1}{4}$ & $n^{\sigma+1 / 4}$ \\
\hline 4. & $"$ & $\begin{array}{c}\text { at the point } \\
x=z\end{array}$ & $\sigma>-\frac{1}{2}$ & $n^{\sigma+1 / 4}$ \\
\hline
\end{tabular}

5. Same results hold in case $(z-a)(z-b)=0$ under analogous conditions imposed on $p(x)$ and $\varphi_{n}(x)$.

\begin{tabular}{|c|c|c|c|c|} 
6. & & $\begin{array}{c}\text { no } \\
\text { conditions }\end{array}$ & $\begin{array}{c}\text { at the point } \\
x=z\end{array}$ \\
7. & any & no conditions & & $n^{\sigma+1 / 2}$ \\
\end{tabular}

The most interesting case is

$$
\sigma=0 ; \quad\left|r_{r, f}(z)\right|< \begin{cases}\tau E_{n}(f) \log n & \text { (under conditions }(12,13)) \\ \tau E_{n}(f) n^{1 / 4} & \text { (under conditions }(12,18)) \\ \tau E_{n}(f) n^{1 / 2} & \text { (under condition (18)) }\end{cases}
$$

The condition (18) with $\sigma=0$ holds, for instance, in the case of the characteristic function (8) (see second footnote on page 540) at any point $x=z[(z-a)(z-b) \neq 0]$, where $\Pi(z) \neq 0$.

Another case, where we have (18) satisfied with $\sigma=0$, is given by G. Szegö.+

It remains to prove that it is impossible to have $(12,13)$ with $\sigma<0$ (see Table B, case 1).

In fact, the contrary assumption gives

(27) $\frac{r_{n, f}(x)}{n^{\sigma}} \rightarrow 0$ for $n \rightarrow \infty$ uniformly $\quad\left(\sigma<0 ; c<c^{\prime} \leqq x \leqq d^{\prime}<d\right)$, since $E_{n}(f) \rightarrow 0$ with $1 / n$ for every continuous function.

Writing in general $E_{n}(f ; a, b)$, we get, from the very definition of this quantity,

$$
E_{n}\left(f ; c^{\prime}, d^{\prime}\right) \leqq \max \left|r_{n, f}(x)\right| \text { for } \quad c^{\prime} \leqq x \leqq d^{\prime} .
$$

${ }^{*} \tau$ is a fixed constant, not depending on $n$, nor on $z$ (see $(12,13)$ ).

$\dagger$ In some cases we know the order of $K_{n}(z)$, but not that of $\varphi_{n}(z)$.

$\ddagger$ G. Szegö, Über den asymptotischen Ausdruck von Polynomen, Mathematische Annalen, vol. 86 (1922), pp. 114-140; p. 139. 
Therefore, according to (27),

$$
E_{n}\left(f ; c^{\prime}, d^{\prime}\right)=o\left(n^{\sigma}\right) \text { with } \sigma<0,
$$

for every continuous function, which is impossible, because, as was established by D. Jackson, ${ }^{*}$ for any $\sigma<0$ there always exists a continuous function $f(x)$, for which

$$
\frac{E_{n}\left(f ; c^{\prime}, d^{\prime}\right)}{n^{\sigma}} \rightarrow 0 \text { for } n \rightarrow \infty \text {, Q. E. D. } \dagger
$$

4. Order of $\varphi_{n}(x)$ (with respect to $n$ ). $\ddagger$ We modify slightly our notations for Tchebycheff's polynomials and write in general, the characteristic function being $p(x)$,

$$
\varphi_{n}(p ; x)=a_{n}(p) x^{n}+\ldots \quad\left(n=0,1,2, \ldots ; a_{n}(p)>0\right) .
$$

We shall proceed to compare $\varphi_{n}(p ; x)$ and $\varphi_{n}(q ; x)$ corresponding to the same interval $(a, b)$.

For this purpose consider

$$
\begin{aligned}
\Delta_{p, q} & =\int_{a}^{b} q(x)\left[\varphi_{n}(q ; x)-\varphi_{n}(p ; x)\right]^{2} d x=i_{1}-2 i_{2}+i_{3}, \\
i_{1} & =\int_{a}^{b} q(x) \varphi_{n}^{2}(q ; x) d x=1, \\
i_{2} & =\int_{a}^{b} q(x) \varphi_{n}(p ; x) \varphi_{n}(q ; x) d x=\frac{a_{n}(p)}{a_{n}(q)}, \\
i_{3} & =\int_{a}^{b} q(x) \varphi_{n}^{2}(p ; x) d x=1+\int_{a}^{b}[q(x)-p(x)] \varphi_{n}^{2}(p ; x) d x \\
& =1+\theta_{1}\left(\frac{q-p}{p}\right)_{\max } \quad\left(\left|\theta_{1}\right| \leqq 1\right),
\end{aligned}
$$

where in general $(u)_{\max },(u)_{\min }$ stand for the least upper and greatest lower bound respectively (or maximum and minimum) of $|u(x)|$ in $(a, b)$.

We make use now of following inequalities:

$$
\left(\frac{q}{p}\right)_{\min }<\frac{a_{n}^{2}(p)}{a_{n}^{2}(q)}<\left(\frac{q}{p}\right)_{\max }, \S
$$

* Loc. cit., p. 56.

$\dagger$ But we may have $\left|\varphi_{n}(x)\right|<\tau n^{\sigma}$ with $\sigma<0$ at a certain point $x=z$; e. g., for the polynomials of Jacobi (p. 540), with $\alpha, \beta<\frac{1}{2}$ at $x=a, b\left(\sigma=\alpha-\frac{1}{2}, \beta-\frac{1}{2}\right.$, respectively).

$\ddagger$ The results of this paragraph are summarized in my article Sur les polynomes de Tchebycheff, Com p tes Rendus, vol.178 (1924), p.2229. Here they are somewhat generalized.

$\S$ J. Chokhate, Sur quelques propriétés des polynomes de Tchebycheff, Comptes Rendus, vol. 166 (1918), pp. 28-30. 
which give

$$
\left|\frac{a_{n}(p)}{a_{n}(q)}-1\right|<\left(\frac{q-p}{p}\right)_{\max } .
$$

Thus we have, using (29),

$$
\begin{aligned}
i_{2} & =1+\theta_{2}\left(\frac{q-p}{p}\right)_{\max } & & \left(\left|\theta_{\mathbf{2}}\right| \leqq 1\right) \\
\Delta_{p, q} & =\delta_{n}\left(\frac{q-p}{p}\right)_{\max } & & \left(0<\delta_{n}<3\right) .
\end{aligned}
$$

On the other hand, $Q_{n}(x)$ being an arbitrary polynomial of degree $\leqq n$, we have

$$
\begin{aligned}
Q_{n}(x) & =\sum_{0}^{n} A_{i} \varphi_{i}(q ; x), \quad A_{i}=\int_{a}^{b} q(x) Q_{n}(x) \varsigma_{i}(q ; x) d x, \\
\left|Q_{n}(x)\right| & \leqq \sqrt{\sum_{0}^{n} A_{i}^{2}} \sqrt{\sum_{0}^{n} \varphi_{i}^{2}(q ; x)}
\end{aligned}
$$

$$
\left|Q_{n}(x)\right| \leqq \sqrt{\int_{a}^{b} q(x) Q_{n}^{2}(x) d x} \sqrt{K_{n}(q ; x)}, K_{n}(q ; x) \equiv \sum_{0}^{n} \varphi_{i}^{2}(q ; x) .
$$

Apply (32) to the polynomial $\varphi_{n}(p ; x)-\varphi_{n}(q ; x)$ and use (31). We get

$$
\left.\begin{array}{l}
\left|\varphi_{n}(p, x)-\varphi_{n}(q ; x)\right|<v \sqrt{\left(\frac{q-p}{p}\right)_{\max }} \sqrt{K_{n}(q ; x)} \\
\left|\varphi_{n}(p ; x)-\varphi_{n}(q ; x)\right|<v \sqrt{\left(\frac{q-p}{q}\right)_{\max }} \sqrt{K_{n}(p ; x)}
\end{array}\right\} \quad(0<\tau<\sqrt{\mathbf{3}})
$$

Formulas $(30,33)$ lead to following

THEOREM II. Suppose that $q(x)$, containing a parameter $\alpha$, tends for $a \rightarrow \alpha_{0}$ to $p(x)$ uniformly in $(a, b)$, and that $p(x) \geqq p_{\min }>0$ in $(a, b)$. Then $\varphi_{n}(q ; x) \rightarrow \varphi_{n}(p ; x)$ uniformly in $(a, b)$, and $a_{n}(q)$ under the above conditions tends uniformly (with respect to $n$ ) to $a_{n}(p)$.

Proof. $\varepsilon$ being chosen as small as we please, take $\left|\alpha-\alpha_{0}\right|$ sufficiently small in order to give, for $a \leqq x \leqq v$,

$$
\begin{aligned}
& |q(x)-p(x)|<\frac{p_{\min }}{2}, \\
& |q(x)-p(x)|<\frac{\varepsilon^{2} p_{\min }}{6 K_{n}}, \\
& |q(x)-p(x)|<\varepsilon p_{\min }, \quad \text { for } a \leqq x \leqq b,
\end{aligned}
$$


where $K_{n}=\max K_{n}(p ; x)$ in $(a, b)$. Then

$$
\begin{aligned}
& q_{\min }>\frac{p_{\min }}{2}, \quad\left(\frac{q-p}{p}\right)_{\max }<\frac{2(q-p)_{\max }}{p_{\min }}, \\
& \left|\varphi_{i}(p ; x)-\varphi_{i}(q ; x)\right|<\varepsilon \quad(i=0,1, \cdots, n ; a \leqq x \leqq b), \\
& \left|\frac{a_{n}(p)}{a_{n}(q)}-1\right|<\varepsilon \text { for every } n, \quad \text { Q. E. D. }
\end{aligned}
$$

Consider first two special systems of Tchebycheff's polynomials:

$$
\begin{aligned}
& \varphi_{n}(p ; x) \text { with } p(x)=(x-a)^{\alpha-1}(b-x)^{\beta-1} \Pi(x) \\
& (\alpha, \beta>0 ; \Pi(a) \Pi(b) \neq 0) ; \Pi(x) \text { a polynomial of degree } s \\
& \varphi_{n}(q ; x) \text { with } q(x)=(x-a)^{\alpha-1}(b-x)^{\beta-1}, \\
& (\alpha, \beta>0) \text { (polynomials of Jacobi). }
\end{aligned}
$$

We have used these polynomials above (see pages 540, 544). We are now interested in finding what are the relations between $\varphi_{n}(1 ; x), K_{n}(p ; x)$ and the degree $s$ of $\Pi(x)$ in (34).

For this purpose consider the development

(36) $\Pi(x) \varphi_{n}(p ; x)=\sum_{0}^{n+s} A_{i} \varphi_{i}(q ; x), \quad A_{i}=\int_{a}^{b} p(x) \varphi_{n}(p ; x) \varphi_{i}(q ; x) d x$, where, as we see immediately,

$$
A_{0}=A_{1}=\ldots=A_{n-1}=0 .
$$

On the other hand, as is well known,

$$
\begin{gathered}
\left|\varphi_{n}(q ; z)\right|<\boldsymbol{\tau} \quad(a+\varepsilon \leqq z \leqq b-\varepsilon), \\
\left|\varphi_{n}(q ; a)\right|<\tau n^{\alpha-1 / 2}, \quad\left|\varphi_{n}(q ; b)\right|<\tau n^{\beta-1 / 2},
\end{gathered}
$$

where $\tau$ does not depend on $z$, nor on $n$.

Hence, $(36,37)$ give

$$
\begin{aligned}
\Pi(x)\left|\varphi_{n}(p ; x)\right| & \leqq \sqrt{\int_{a}^{b} p(x) \Pi(x) \varphi_{n}^{2}(p ; x) d x} \sqrt{\sum_{n}^{n+s} \varphi_{i}^{2}(q ; x)} \\
& \leqq \sqrt{\Pi_{\max }} \sqrt{\sum_{n}^{n+s} \varphi_{i}^{2}(q ; x)} .
\end{aligned}
$$

Using (38), we get, $n$ being sufficiently large (since $\varphi_{n}(q ; x)$ does not depend on $s$ ): 


$$
\begin{aligned}
& I(z)\left|\boldsymbol{\varphi}_{n}(p ; z)\right|<\boldsymbol{v} \widehat{s} \quad(a+\varepsilon \leqq z \leqq b-\varepsilon), \\
& \left|\varphi_{n}(p ; z)\right|<\tau \sqrt{s} \quad((a<) c+\varepsilon \leqq z \leqq d-\varepsilon(<b) ; \Pi(x) \neq 0 \text { in }(c, d)), \\
& \left|\varphi_{n}(p ; a)\right|<\tau V \bar{s} n^{\alpha-1 / 2} ; \quad\left|\varphi_{n}(p ; b)\right|<\tau V_{s}^{-} n^{\beta-1 / 2},
\end{aligned}
$$

where $\tau$ does not depend on $z$, nor on $n$, nor on $s$.

Formulas (40) answer the question stated above.

We return now to the general case. Assume that there exists a certain interval $(c, d)$ such that

(41) $p(x)$ is continuous and positive for $c \leqq x \leqq d(a \leqq c ; d \leqq b)$.

Consider the polynomial $T_{m, p}(x)$ of best approximation to $p(x)$ in $(c, d)$ of sufficiently large degree $m$. The polynomial $T_{m, p}(x)$ is also positive in $(c, d)$. Now introduce the functions $\varphi_{n}(q ; x)$, with

$$
\begin{aligned}
q(x) & \equiv T_{m, p}(x) \text { in }(c, d) \\
& \equiv p(x) \text { in }(a, c) \text { and }(d, b) .
\end{aligned}
$$

We can apply (33), which gives (since $\left.(q-p)_{\max }=-E_{m}(p)\right)$

$$
\left|\varphi_{n}(p ; x)-\varphi_{n}(q ; x)\right|<\tau \sqrt{E_{m}(p) K_{n}(p ; x)},
$$

where $\tau$ does not depend on $x$, nor on $n$, nor on $m .^{*}$

We assume that $m$ and $n$ are increasing indefinitely, but $m / n \rightarrow 0$. Formula (43) combined with (40) (where $p(x), s, a, b$ must be replaced respectively by $q(x), m, c, d$, and $\alpha=\beta=1$, since $q(x)>0$ for $c \leqq x \leqq d$ ) and with the results of Theorem I (page 540) gives, $m$ and $n$ being sufficiently large, the fundamental formula

$$
\begin{gathered}
\left|\varphi_{n}(p ; z)\right|<\boldsymbol{\tau}\left[\sqrt{m}+\sqrt{n E_{m}(p)}\right] \quad(c+\varepsilon \leqq z \leqq d-\varepsilon), \\
\left|\varphi_{n}(p ; c)\right|,\left|\varphi_{n}(p ; d)\right|<\tau \sqrt{n}\left[\sqrt{m}+\sqrt{n E_{m}(p)}\right]
\end{gathered}
$$

under condition (41), where $\tau$ does not depend on $z$, nor on $n$, nor on $m$, and $\varepsilon>0$ is arbitrarily small, but fixed.

In order to derive from (44) all the conclusions available, we take

$$
m=\text { integral part of } n^{\beta} \text { with } 0<\beta<1, n \rightarrow \infty \text {. }
$$

* Formula (43) holds also for $(a, b)$ infinitely large, provided $(c, d)$ is finite. 
Then

$$
m \rightarrow \infty, n \rightarrow \infty, \frac{m}{n} \rightarrow 0, \frac{m}{n^{\beta}} \rightarrow 1, E_{m}(p) \rightarrow 0
$$

We can now use Table A (page 539), $\beta$ being chosen so as to make the right-hand member in (44) of the highest order possible with respect to $1 / n$. The results thus obtained are summarized in the following Table.

Table C: $\varphi_{n}(p ; x)$

\begin{tabular}{|c|c|c|}
\hline Conditions imposed on $p(x)$ & $z$ & $\varphi_{n}(p ; z)=$ \\
\hline $\begin{array}{l}\text { 1.p } p(x) \text { is continuous and positive for } \\
(a \leqq c \leqq x \leqq d(\leqq b)\end{array}$ & $\begin{array}{c}c+\varepsilon \leqq z \\
\leqq d-\varepsilon\end{array}$ & $o\left(n^{1 / 2}\right)$ \\
\hline $\begin{array}{l}\text { 1, and 2. }\left|p^{(k)}\left(x_{2}\right)-p^{(k)}\left(x_{1}\right)\right|<\lambda\left|x_{2}-x_{1}\right|^{\alpha} \\
\quad \text { for } c \leqq x_{1}, x_{2} \leqq d ; p^{(0)}(x) \equiv p(x)\end{array}$ & $=$ & $O\left(n^{1 / 2(1+k+\alpha)}\right)^{*}$ \\
\hline $\begin{array}{l}\text { (in particular for } k=0, \alpha=1: \text { Lipschitz } \\
\text { condition). }\end{array}$ & $"$ & $O\left(n^{1 / 4}\right)$ \\
\hline 1, and 3. $p^{(k)}(x)$ is continuous for $c \leqq x \leqq d$. & थ & $O\left(n^{1 / 2(1+k)}\right)$ \\
\hline $\begin{array}{l}\text { 1, and 4. } p(x) \text { is indefinitely differentiable in } \\
\quad(c, d) \text {. }\end{array}$ & $"$ & $\begin{array}{c}o\left(n^{\sigma}\right), \sigma>0 \\
\text { arbitrarily } \\
\text { small }\end{array}$ \\
\hline 5-8. Same conditions as in 1-4 above. & $\begin{array}{l}a=c \leqq z \\
\leqq l=b\end{array}$ & $\begin{array}{l}1 / 2 \text { must be } \\
\text { added to each } \\
\text { of the expo- } \\
\text { nents of } n \text { in } \\
1-4 \text { above. }\end{array}$ \\
\hline
\end{tabular}

The Tables A, B, C, as well as the results of $\S 2$ (concerning $K_{n}(p ; x)$ ) enable us to determine the convergence and the order (with respect to $n$ ) of the remainder of the development of a continuous function into a series according to Tchebycheff's polynomials of a given type.

Many theorems can be formulated in this way. As an illustration, we state the following:

Theorem III. Suppose $f(x)$ is continous in a given finite interval $(a, b)$ and satisfies the condition

$$
\left|f\left(x_{2}\right)-f\left(x_{1}\right)\right| \leqq \omega(\delta) f o r \cdot\left|x_{1}-x_{2}\right| \leqq \delta \quad\left(a \leqq x_{1}, x_{2} \leqq b\right)
$$

* See G. Szegö, loc. cit., p. 139. 
Then, in the development

$$
\begin{gathered}
f(x)=\sum_{0}^{n} A_{i} \varphi_{i}(x)+r_{n, f}(x), \quad A_{i}=\int_{a}^{b} p(x) f(x) \varphi_{i}(x) d x, \\
\text { where } \quad(x)=(x-a)^{\alpha-1}(b-x)^{\beta-1} \Pi(x) \quad(\alpha, \beta>0), \\
\Pi(x) \text { a polynomial }(\Pi(a) \Pi(b) \neq 0),
\end{gathered}
$$

we have

$$
r_{n, f}(z)=O\left(\omega\left(\frac{b-a}{n}\right) \log n\right)^{*}
$$

at any point $x=z$ inside $(a, b)$, provided $\Pi(z) \neq 0 . \quad$ In particular, the development under consideration converges to $f(x)$ uniformly for $c+\varepsilon \leqq x \leqq d-\varepsilon(a \leqq c ; d \leqq b ; \varepsilon>0$ arbitrarily small, but fixed), if $f(x)$ satisfies a Dini-Lipschitz condition, provided the interval $(c, d)$ contains no roots of $\boldsymbol{\Pi}(x)$.

In the particular case $\alpha=\beta=1, \Pi(x) \equiv 1$, we get the results obtained by D. Jackson, as was mentioned above.

* This follows from Table $A_{1}$, Table $B_{2}$ and p. 544.

The University of Chicago,

Chicago, Ill. 